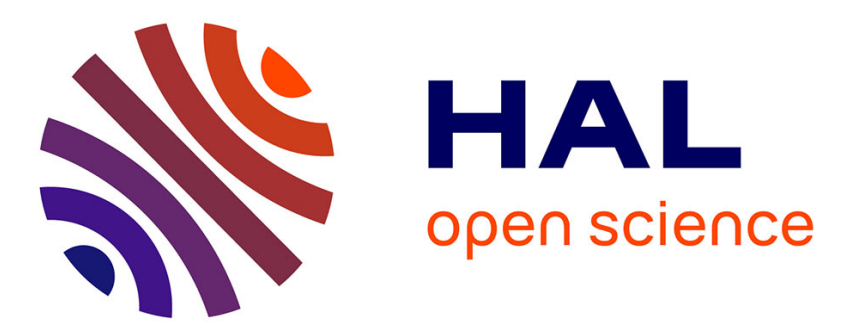

\title{
WHAT IS KNOWN EXPERIMENTALLY ON SPIN AND MOMENTUM WAVE FUNCTIONS IN NEUTRON BEAMS
}

F. Mezei

\section{- To cite this version:}

F. Mezei. WHAT IS KNOWN EXPERIMENTALLY ON SPIN AND MOMENTUM WAVE FUNCTIONS IN NEUTRON BEAMS. Journal de Physique Colloques, 1984, 45 (C3), pp.C3-223-C3-228. 10.1051/jphyscol:1984337 . jpa-00224051

\section{HAL Id: jpa-00224051 https://hal.science/jpa-00224051}

Submitted on 1 Jan 1984

HAL is a multi-disciplinary open access archive for the deposit and dissemination of scientific research documents, whether they are published or not. The documents may come from teaching and research institutions in France or abroad, or from public or private research centers.
L'archive ouverte pluridisciplinaire HAL, est destinée au dépôt et à la diffusion de documents scientifiques de niveau recherche, publiés ou non, émanant des établissements d'enseignement et de recherche français ou étrangers, des laboratoires publics ou privés. 


\title{
WHAT IS KNOWN EXPERIMENTALLY ON SPIN AND MOMENTUM WAVE FUNCTIONS IN NEUTRON BEAMS
}

\author{
F. Mezei \\ Institut Laue-Langevin, 156X, F-38042 Grenoble Cedex, France
}

\begin{abstract}
Résumé - II n'y a pas de méthode expērimentale connue pour la détermination des Fonctions d'onde spatiales des neutrons dans un faisceau. Par contre, il est aisé de déterminer. la fonction d'onde dominante des états du spin. dans un faisceau polarisé. Cela rend possible 1'observation de phênomènes spécifiques aux fonctions d'onde dans 1 'espace de la variable de spin, comme dans les exemples présentës dans ce papier. La relation entre fonctions d'onde dans les espaces du spin et du moment cinétique sera aussi examinée.

Abstract - There is no known experimental method to determine the spatial wave functions of the neutrons in a beam. To the contrary, it is simple matter to determine the dominant spin state wave function in a polarized beam. This allows the observation of typical coherent wave function phenomena in the spin variable space, examples of which are described. The relation between spin and momentum space wave functions is also considered.
\end{abstract}

In the spirit of quantum mechanics we have to assume that the particles in a beam are represented by an ensemble a wave functions $\psi_{i}=\int a_{i}(\vec{k}) \exp (i \vec{k} r) d \vec{k}$ most naturally thought of as being wave packets. For many practical purposes, however, a less complete knowledge of the particle states in the beam suffices, namely the one provided by a classically conceived momentum (velocity) distribution function

$$
p(\vec{k})=\left\langle\left|a_{i}(\vec{k})\right|^{2}\right\rangle_{i}
$$

(where $<>_{i}$ stands for the average over all particles $i$ ). Taking into account neutron spin, we should define $p_{\uparrow}(\vec{k})$ and $p_{\downarrow}(\vec{k})$ for the two spin $1 / 2$ eigenstates with respect to some quantization axis. For the moment, in agreement with most practical situations, we will assume that the momentum and spin distributions are independent, therefore we can describe the spin distribution by two probabilities $p_{\uparrow}$ and $p_{\downarrow}$. Thus the spin wave functions can be considered as $x_{i}=\alpha_{i}\left|\uparrow>+\beta_{i}\right| \downarrow>$, and

$$
\mathrm{p}_{\uparrow}=\left\langle\alpha_{i}{ }^{*} \alpha_{i}\right\rangle_{i} \quad \mathrm{p}_{\downarrow}=\left\langle\beta^{*}{ }_{i} \beta_{i}\right\rangle_{i}
$$

The only difference between the probability distribution in momentum and spin spaces come from the dimensionality of the corresponding Hilbert spaces, respectively infinite and two.

Specific information on wave functions, i.e. going beyond the probability distribution functions (1) and (2), means the knowledge of phase relations between the coefficients in the above expressions of the wave functions. This implies (a) that the off-diagonal averages of the type

$$
<a_{i}^{*}\left(k^{\prime}\right) a_{i}(k)>_{i} \quad \text { or } \quad\left\langle\alpha_{i}^{*}{ }_{i}{ }^{\prime} i\right.
$$

are not identically zero (coherence) and (b) that we have access to them by controlling the beam preparation and/or by directly measuring them for a given beam. While there is no known way of determining these off-diagonal averages for the momentum distribution, they can be easily controlled and measured for the spin variable. (Note that the averages in eqs. (1-3) together form the density matrices). 
Indeed, scattering cross sections only depend /1/ on the distribution functions (1) and (2), and thus they do not offer any possibilities of determining the crossterms (3). In fact, in some cases it can be known that the scattered beam corresponds to specific wave functions ( $i . e$. there are non-zero cross-terms), nevertheless scattering on an analyser sample does not allow to observe this fact. (An example is the polarization behaviour of paramagnetic scattering, which was theoretically predicted more than 40 years ago, but it could only recently be experimentally demonstrated /2/).

What made the situation evolve concerning the spin states, is the development of spin turn devices $/ 3,4 /$ which allow the physical transformation of any given spin wave function into any other. Since by essentially seattering type processes we are able to produce beams with e.g. nearly exclusively $|\uparrow\rangle$ states $\left(p_{\uparrow} \sim 1, p_{\uparrow} \simeq 0\right.$ ), or for the same matter, select and analyse such states in a beam, this gives us full access to the whole of the spin 1/2 Hilbert space. Namely, e.g. the x-component of the spin polarization (which can be measured by turning it into the z-quantization direction of the analyser) is given as

$$
P_{x}=\left\langle\sigma_{x}\right\rangle_{i}=\left\langle\alpha_{i}^{*} \beta_{i}+\beta_{i}^{*} \alpha_{i}\right\rangle_{i}
$$

This, together with $\mathrm{P}_{\mathrm{y}}$, allows the determination of the off-diagonal averages in (3). (Since no practical device is perfect, we can never produce a beam with all neutrons being in the same spin state $\chi$. However, we can produce a situation equivalent of having a large fraction of the beam in one state, and the rest being uniformly distributed over the spin variable space. The actual distribution of $x_{i}^{\prime} s$ in a beam, can of course never be known or measured, cf. for example Ref. /5/). The experimental determination of $P_{X}$ is technically complicated by the neutron velocity spread in the beam, since it leads to a dephasing of the Larmor precessions. Therefore either sufficiently monochromatic beams or neutron spin echo refocussing has to be applied $/ 3 /$.

\section{WAVE FUNCTION PHENOMENA : COHERENT SCATTERING AND SPIN SUPERPOSITION}

The simplest wave function phenomenon in the spin $1 / 2$ variable space is the observation of the evolution of the spin state in a magnetic field $\vec{B}$ (Larmor precession). The quantum behaviour in this respect, however, happens to coincide exactly with the classical one, the relation between the classical spin direction vector $S$ with polar angles $\theta, \phi$ and the corresponding spin wave function $x$ being (see e.g. Ref./5/)

$$
|x>=\alpha| \uparrow>+\beta|\psi\rangle=\cos \frac{\theta}{2}|\uparrow\rangle+e^{i \phi} \sin \frac{\theta}{2}|\downarrow\rangle
$$

However, the two effects (whose experimental verification is described below for the first time) have no obvious classical analogs, and they correspond to the extension of the wave function analysis (as opposed to consideration of incoherent probabilities $p_{\uparrow}$ and $p_{\downarrow}$ only) to scattering objects, in particular polarizers. The change of the neutron spin state due to interaction with any homogeneous system can be described by an $\overrightarrow{\mathrm{S}}$ matrix

$$
\left|x^{\prime}\right\rangle=\left[\begin{array}{l}
\alpha^{\prime} \\
\beta^{\prime}
\end{array}\right]=\left[\begin{array}{ll}
a_{\uparrow \uparrow} & a_{\uparrow \downarrow} \\
a_{\downarrow \uparrow} & a_{\downarrow \downarrow}
\end{array}\right]\left[\begin{array}{l}
\alpha \\
\beta
\end{array}\right]=\hat{s}|x\rangle
$$

The homogeneity of the system in both space and time allows us to assume that both the old and new spin states, $\mid \chi^{\rangle}$and $\left|x^{\prime}\right\rangle$, are independent from space and time, i.e. separable from the spatial wave function. A priori the $\hat{S}$ matrix shows no symmetry, (e.g. $\left|a_{\uparrow \downarrow}\right| \neq\left|a_{\downarrow \uparrow}\right|$ ) and eq. (6) only"expresses the basic postulated linearity of quantum theory. All 4 complex elements of the $\hat{S}$ matrix can be determined (within a common arbitrary phase factor) in a straightforward manner by measuring the $\mathrm{x}, \mathrm{y}, \mathrm{z}$ components of the scattered beam polarization $\overrightarrow{\mathrm{P}}^{t}$ for various directions of theincoming polarization $\overrightarrow{\mathrm{P}}=\langle\vec{\sigma}\rangle_{i}$. This gives, for example, 


$$
\left\langle x^{\prime}\left|\sigma_{x}\right| x^{\prime}\right\rangle=\overline{a_{\uparrow \uparrow}^{\star} a_{\downarrow \uparrow}} \alpha_{\alpha}^{*}+\overline{a_{\uparrow \uparrow}^{*} a_{\downarrow \downarrow}} \alpha^{*} \beta+\ldots
$$

where the bar stands for averaging over the scattering system in order to take into account its eventual inhomogeneities in space and time. (Note, that without allowing for this variation of the $\hat{\mathrm{S}}$ matrix in space and time, we would always obtain $\left|\vec{P}^{\prime}\right|=1$ if $|\overrightarrow{\mathrm{P}}|=1$ ). Obviously, terms of the form $\left|a_{\mathrm{k} 1}\right|^{2}$ describe the (incoherent) probabilities that a neutron originally in spin state 1 ends up in state $k$. The wave function (coherence) aspect of the scattering is related to the terms of the type shown in eq. (7) i.e. $\bar{a}_{k}^{*} \bar{a}_{\mathrm{mn}}$ with $\mathrm{k} \neq \mathrm{m}$ and/or $1 \neq \mathrm{n}$.

Table I gives examples of measured values of $\overline{a_{k}^{*}} \overline{a_{m n}}$ for a total reflection mirror and a supermirror type $/ 6 /$ polarizer in various magnetizing fields. The fact that $\mathrm{R}=\left|\overrightarrow{\mathrm{a}_{\uparrow \uparrow}}\right| 2 /\left|\overrightarrow{a_{\downarrow \downarrow}}\right|^{2} \gg 1$ indicates that there is a strong polarizing action. The substantial value of $\left|\overrightarrow{a_{\uparrow \uparrow}^{*} a_{\downarrow \uparrow}}\right|$ (compared with $\left(\left|\overline{a_{\uparrow \uparrow}}\right| 2|\overrightarrow{a \downarrow \uparrow}| 2\right) 1 / 2=0.30$ ) in the last row of the table is evidence for the partial coherence between the "$\uparrow$ to $\uparrow$ " and " $\uparrow$ to $t$ " processes, which makes an originally $100 \%$ polarized beam parallel to $\vec{B}$ leave the polarizer with $85 \%$ polarization in a direction which makes an angle of $29^{\circ}$ with the $\uparrow$ direction. This is in contrast to the conventional incoherent picture, in which a polarizer is supposed to always produce a polarization parallel to the field direction. Note that this coherence between the $a_{\uparrow \uparrow}$ and $a_{\downarrow \uparrow}$ terms disappears at higher magnetic fields.

Another wave function phenomenon is the coherent superposition of spin states. As it can be seen from eq. (5), the superposition of $\uparrow$ and $\downarrow$ spin states with amplitudes $\alpha$ and $\beta$, respectively, produces a pure state with a spin vector pointing in a direction at an angle $\theta$ with respect to the quantization axis, where $\operatorname{tg} \theta / 2$ $|\beta| /|\alpha|$. (This means that this state would be $\uparrow$ if the quantization axis were chosen to be the direction $\theta, \phi)$. This unusual behaviour can be checked by changing the ratio $|\beta| /|\alpha|$, which is simply achieved by a polarizer device with $\left|\overline{a_{\uparrow \uparrow}}\right| /\left|\overline{a_{\downarrow \downarrow}}\right|=$ $\sqrt{\mathrm{R}} \neq 1$, cf. eq. (6). Thus the angle $\theta^{\prime}$ between the field direction $(\uparrow)$ and the spin vector of the neutron after the polarizer is given as $/ 6 /$

$$
\operatorname{tg} \frac{\theta^{\prime}}{2}=\frac{1}{\sqrt{R}} \operatorname{tg} \frac{\theta}{2},
$$

if the off-diagonal elements of the $\hat{\mathrm{S}}$ matrix can be neglected (which can be shown to be the casé if $\pi-\theta \geq 10^{\circ}$, and $\left|\overline{a_{\uparrow \downarrow}}\right| 2,\left|\frac{\mid a_{\downarrow}}{a^{\prime}}\right|^{2}<0.01$ ) and the $a_{\uparrow \uparrow}$ and $a_{\downarrow \downarrow}$ elements

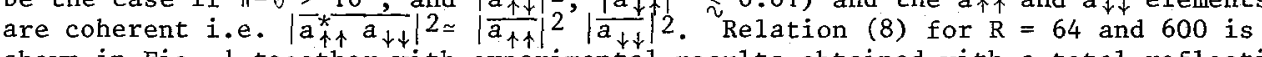
shown in Fig. 1 together with experimental results obtained with a total reflecting mirror and a supermirror in a magnetizing field of 800 øe. The good agreement between the theoretical and measured curves shows the coherent character of the superposition of the $\uparrow$ and $\psi$ spin states.

\section{WAVE FUNCTIONS AND INTERFERENCE}

Interference experiments represent a less complete approach to the investigation of coherent superposition of states. They do not allow the determination of wave functions, i.e. to establish the coherence between the different components in the eigenstate expansion, $c f$. eq. (3). What is being done instead in interference studies, is to split the beam physically (e.g. by a half-transparent mirror) and study the coherence in the recombination of the two parts. This is in fact a test of the superposition principle within single solutions of the equation of motion through the interferometers, which solutions correspond to various $k$ eigenstates before the interferometer. In practice, the coherence of the beam splitting is observed by introducing a variable phase difference between the two split components before their recombination. The simplest interference phenomenon is the neutron spin Larmor precession, in which the phase difference between the $\uparrow$ and $\downarrow$ components of a partial wave with initial momentum $k$ is produced by the Zeeman energy splitting $\pm \mu \mathrm{B}$ (Fig. 2). The interference pattern obtained is the simple (incoherent) sum of the results one expects for the various $k$ components in the beam (i.e. the 


\begin{tabular}{|c|c|c|c|c|c|c|}
\hline $\begin{array}{l}\text { Polarizer } \\
\text { applied }\end{array}$ & and field & $\sqrt{a_{\uparrow \uparrow}}$ & $\left|\overline{a_{\uparrow \downarrow}}\right|^{2}$ & $\left|\overline{a_{\downarrow \uparrow}}\right|^{2}$ & $\left|\overline{a_{\downarrow \downarrow}}\right|^{2}$ & $\left|\overline{a_{\uparrow \uparrow}^{*}} \bar{a}_{\uparrow \uparrow}\right|^{2}$ \\
\hline TR mirror, & $170 \emptyset \mathrm{e}$ & $\sim 1$ & .017 & .011 & .028 & - \\
\hline $\begin{array}{c}\text { SM mirror, } \\
" 1 \\
"\end{array}$ & $\begin{array}{l}1200 \emptyset \mathrm{e} \\
170 \emptyset \mathrm{e} \\
5 \emptyset \mathrm{e} \\
1 \emptyset \mathrm{e}\end{array}$ & $\begin{array}{l}.87 \\
.87 \\
.85 \\
.82\end{array}$ & $\begin{array}{l}.014 \\
.016 \\
.038 \\
.120\end{array}$ & $\begin{array}{l}.008 \\
.009 \\
.033 \\
.113\end{array}$ & $\begin{array}{l}.002 \\
.002 \\
.004 \\
.013\end{array}$ & $\begin{array}{c}<.001 \\
<.001 \\
- \\
.165\end{array}$ \\
\hline
\end{tabular}

Table I. Measured average products of various elements of the $\hat{\mathrm{S}}$ matrix for a total reflecting (TR) mirror and a supermirror (SM) polarizer in various magnetizing fields.
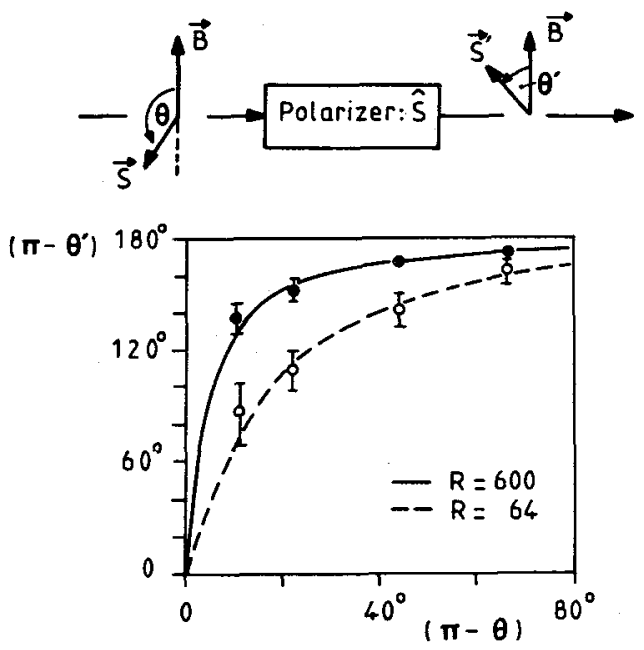

Fig. 1. - Coherent spin superposition: The change of the angle between the spin direction and the magnetic field due to the action of a supermirror polarizer (filled symbols) and a total reflecting mirror polarizer in 800 Øe applied field. The curves represent the theoretical prediction eq. (8) for the $R$ values indicated. $1.5 \mathrm{\&}$ wavelength, about $1 \%$ monochromatic neutron beam was used, without spin echo Larmor precession focussing.

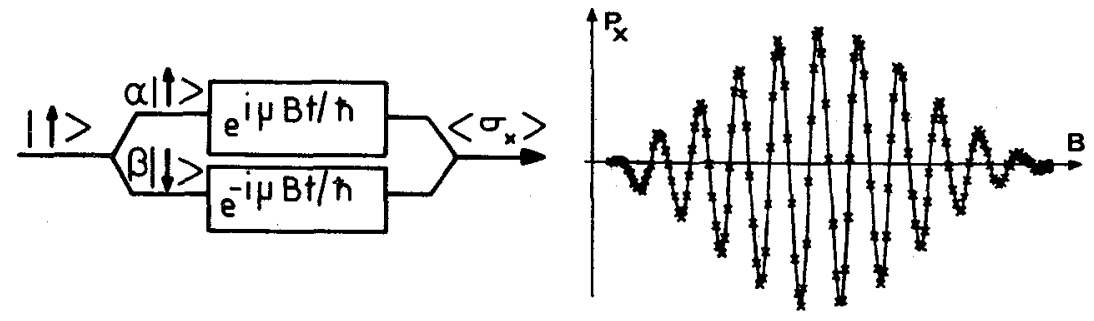

Fig. 2. - Larmor precession as interference. The coherent splitting of the incoming $\uparrow$ state into a superposition of $\uparrow$ and $\downarrow$ states is achieved by a $90^{\circ}$ spin flip, the phase difference between these two components is due to the Zeeman energy as given in eq. (9), and the measurement of $P_{x}$ represents the recombination. The observed wave packet like pattern simply corresponds to the classical neutron velocity distribution in the beam (18\% in the case shown). For details see Refs. $/ 3 /$ and $/ 9 /$. 
Fourier transform of the inverse velocity distribution function /3/).

Thus, in spite of the very convincingly looking wave packet-like results (cf. Fig. 2) interference experiments of this kind (similarly to scattering experiments) reveal nothing of the wave function of the neutrons, i.e. of the relation between amplitudes $a(k)$ and $a\left(k^{\prime}\right)$ with $k \neq k^{\prime}$. This applies to all of the time independent neutron interference studies performed by now, all of which can be fully described by the following simple assumptions : (a) the spin states are given by eq. (5), (b) the phase of the $\uparrow$ and $\downarrow$ spin wave components can be calculated as exp(ikr) with $k$ fulfilling the energy conservation requirement

$$
E=\frac{\hbar^{2} k^{2}}{2 m} \mp \mu B+V=\text { cons } t
$$

all over the trajectory (where $V$ is the nuclear or gravitational potential energy), (c) the final result is the incoherent average over the classical velocity distribution.

In particular neither the neutron's spinor character /7/ nor any coherence length and wave packet structure $/ 8 /$ needs to be invoked in the explanation of interferometry experiments, as it has been already pointed out $/ 9,10 /$.

\section{INTERPLAY BETWEEN SPIN AND MOMENTUM STATES}

The independence of the spin and momentum states we have assumed until now is a perfect approximation in all experiments performed by now. Strictly speaking, however, we should consider wave functions of the form

$$
\psi=|\uparrow\rangle \int a_{\uparrow}(k) e^{i k r} d k+|\psi\rangle \int a_{\downarrow}(k) e^{i k r} d k
$$

and realize, that the measured quantity in polarized neutron experiments is not the spin, but the spin current,

$$
\overrightarrow{\mathrm{P}} \propto\langle\mathrm{J} \vec{\sigma}\rangle
$$

where $J$ is the Ehrenfest current operator and for simplicity we only consider the beam propaǵation in one dimension. It can be shown /5/ that instead of eq. (4) we have

$$
P_{x} \propto<J \sigma_{x}>=\int\left[a_{\uparrow}^{*}\left(k^{\prime}\right) a_{\downarrow}(k)+a_{\downarrow}^{*}(k) a_{\uparrow}\left(k^{\prime}\right)\right] d k
$$

where $k^{\prime}=\left(k^{2}+4 \mu B m / \hbar^{2}\right)^{1 / 2} \simeq k$ if $B$ is small. Thus $P_{X}$ is a measure of the overlap of the $\uparrow$ and $\downarrow$ spatial wave functions and it becomes identically zero when $a_{\uparrow}$ and $a_{\downarrow}$ are orthogonal, e.g. as in a Stern-Gerlach experiment. Thus the Larmor precessions $\left(P_{X} \neq 0\right)$ and the Stern-Gerlach effects are phenomena corresponding to opposite limits in the behaviour (for details cf. Ref. /5/). Note, that in contrast to the interference experiments we have considered above, the overlap of $a_{\uparrow}$ and $a_{\downarrow}$ in eq. (11) is a genuine characteristics of the wave functions involved thus in principle it can be different from the overlap of the $\uparrow$ and + velocity distributions in an ensemble of neutron states (beam). This, however, remains to be demonstrated experimentally.

\section{REMERENCES}

1) see KATZ A., J. Math. Phys. $\frac{7}{7}$ (1966) 1802

2) MEZEI F., J. de Physique 43 (1982) C7-9

3) MEZEI F., Z. Phys. 255 (1972) 146

4) REKVELDT M. Th., J. de Physique 32 (1971) C579

5) MEZEI F., in Imaging Processes and Coherence in Physics, Schlenker M. et a1. editors (Springer Verlag, Heidelberg 1980) p.282 
6) MEZEI F., Comm. Phys. 1 (1976) 81: MEZEI F., DAGLEISH P.A., ibid 2 (1977) 41

7) WERNER S.A., et al.,Phȳs. Rev. Lett. 35 (1975) 1053; RAUCH H. et al. Phys. Lett.A 54 (1975) 425

8) KAISER H., WERNER S.A., GEORGE E.A., Phys. Rev. Lett. 50 (1983) 560;

KLEIN A.G., Opat G.I., HAMILTON W.A., ibid 50 (1983) $5 \overline{63}$

9) MEZEI F., in Neutron Interferometry, BONSE U., RAUCH H., editors (Clarendon Press, Oxford 1979) P. 263

10) COMSA G., Phys. Rev. Lett. 51 (1983) 1105 